\title{
Papillary renal cell carcinoma presenting as a renal abscess
}

\author{
J John, ${ }^{1,2}$ M Locketz, ${ }^{3}$ J Lazarus, ${ }^{1}$ S Sinha ${ }^{1}$ \\ ${ }^{1}$ Division of Urology, Department of Surgery, University of Cape Town / Groote Schuur Hospital, Cape Town, South Africa; \\ ${ }^{2}$ Department of Urology, Frere Hospital / Walter Sisulu University, East London, South Africa; \\ ${ }^{3}$ Division of Anatomical Pathology, National Health Laboratory Service / University of Cape Town, Cape Town, South Africa.
}

Corresponding author: Sunil Sinha(s.sinha@uct.ac.za)

Summary: Renal cell carcinoma (RCC), a highly aggressive and lethal urological malignancy, has a wide variation of signs and symptoms. We report a rare case of papillary RCC (pRCC), presenting as a renal abscess. The knowledge of this association between RCC and a renal abscess is critical to prevent misdiagnosis and/or delayed treatment especially in the elderly and immunocompromised patients.

S Afr J Surg 2019;57(2)

http://dx.doi.org/10.17159/2078-5151/2019/v57n2a2966

A 57-year-old HIV negative non-diabetic male presented with a two-month history of progressive left flank discomfort and constitutional symptoms with significant loss of weight. Two months prior, he was diagnosed and commenced on treatment for pulmonary tuberculosis (TB). On examination the patient was emaciated with an ill-defined tender left flank mass. Apart from a normocytic, normochromic anaemia, laboratory workup was unremarkable. The CT scan of the abdomen and pelvis showed a well defined, heterogeneous, rim enhancing, $3 \mathrm{~mm}$ walled, $10 \times 10 \times 13 \mathrm{~cm}$ (TV x AP x CC) mass, arising from the mid and lower pole of the left kidney with air locules (Figure 1A - C). There was no radiological evidence of intra-abdominal lymphadenopathy. Multiple simple cysts of varying sizes were identified in the right kidney and the liver. A tentative diagnosis of a renal abscess was made. The patient had an incision and drainage that yielded $300 \mathrm{ml}$ of thick, foul smelling pus. In view of the patient's history of pulmonary TB, a biopsy of the abscess wall was taken to rule out genitourinary TB. Salmonella non-Typhi, sensitive to Ciprofloxacin was cultured from the pus. No acid-fast bacilli were observed on Ziehl-Neelsen stain and no mycobacteria were cultured. Histopathological and immunophenotyping analysis of the abscess wall suggested papillary renal cell carcinoma. After resolution of the abscess and a negative metastatic work-up, an open radical nephrectomy was performed. The tumour measured $85 \times 45 \times 45 \mathrm{~mm}$ and had ruptured through the capsule (pT4). The cut section revealed a well circumscribed tumour with a yellow/haemorrhagic appearance with minimal normal renal tissue. The renal vein was uninvolved and an R0 resection achieved. The microscopic and immunophenotype features were in keeping with type I papillary RCC and corresponded with World Health Organisation (WHO)/

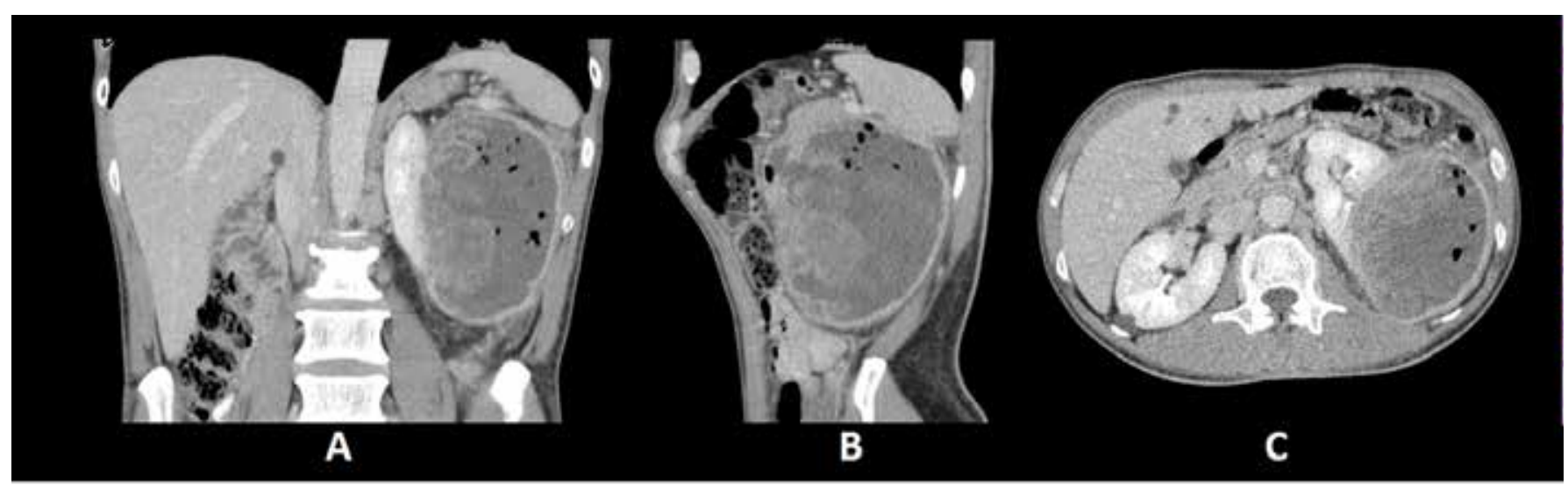

Figure 1A-C. CT scan showing a well defined, heterogeneous, rim enhancing, $3 \mathrm{~mm}$ walled abscess of the left kidney 


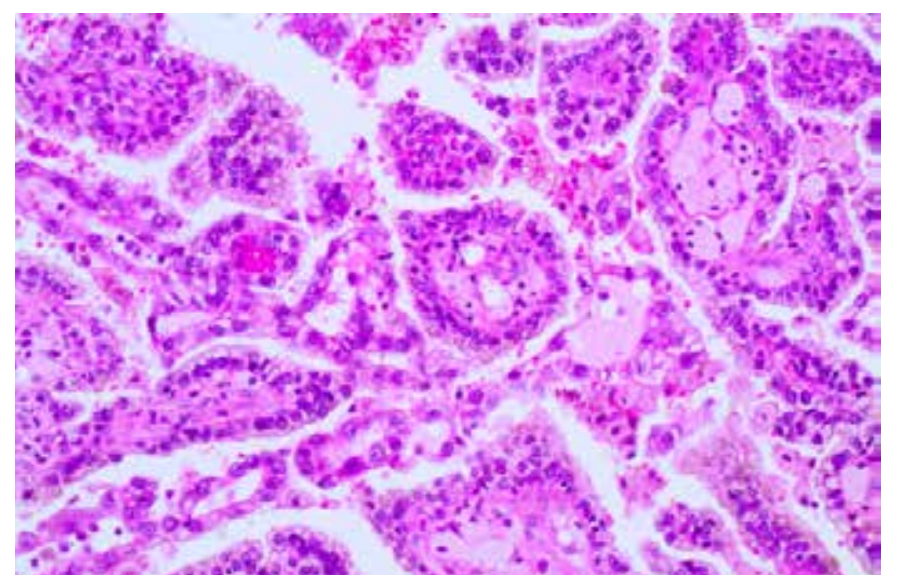

Figure 2. Microscopic features of the mass demonstrating a type I papillary RCC

International Society of Urological Pathology (ISUP) grade 2 (Figure 2). Post nephrectomy, the patient had an uneventful postoperative course.

\section{Discussion}

Renal cell carcinoma (RCC), a highly aggressive and one of the most lethal urological malignancies, accounts for $85 \%$ of all malignant renal neoplasms. With the population ageing and widespread availability of cross sectional abdominal imaging techniques, the absolute incidence of renal cancer still showed an increasing trend over the last few years. In addition, these tumours, which normally presented with the classical triad of flank pain, flank mass and haematuria now have a large variety of presenting features. This report of a papillary RCC (pRCC), masquerading as a renal abscess highlights one of these rarer presentations.

The cross sectional imaging clearly demonstrated air in or adjacent to the renal parenchyma. Apart from recent urological or radiological intervention, the most likely causes of this radiological include perforation of a retroperitoneal abdominal viscus, entero-renal or cutaneo-renal fistula formation, emphysematous pyelonephritis or a renal abscess. A search in medical literature for the association $\mathrm{pRCC}$ with a renal abscess yielded three English language papers. The earliest report from the United Kingdom in 1999 by Olsen et al. had the closest resemblance to our case reporting gas bubbles within the renal parenchyma due to Salmonella species. ${ }^{1}$ In 2018, Mavuduru et al. and Kumar et al. reported further cases of papillary RCC and renal abscess formation from India and the United States respectively without these similarities. ${ }^{2,3}$ Papillary RCCs, which account for $12-15 \%$ of all RCCs, contain cystic degeneration, fibrosis, haemorrhage and necrosis, especially with large sized tumours. Radiologically, they appear hypovascular and heterogeneous due to the prominent central necrosis. This necrosis allows a favourable environment for the proliferation of bacteria, including gas forming Salmonella anaerobe species, which leads to subsequent abscess formation. For clear cell RCC, this tumour necrosis is an adverse pathological factor and is associated with worse clinical outcomes in both uni- and multivariate analyses. The significance of tumour necrosis in non-clear cell RCC is less clear. Renal abscesses are commonly caused by Staphylococci, Escherichia coli, Klebsiella, and Proteus. Urinary tract infections associated with Salmonella non-Typhi are rare and are more commonly seen in infants, patients with structural genitourinary abnormalities or severely immunocompromised patients. Our patient had none of these predisposing factors. The modes of urinary tract infection from Salmonella non-Typhi include haematogenous spread from gastroenteritis or contamination from faecal flora via direct urethral invasion, which is more common in women. ${ }^{4}$

\section{Conclusions}

We report a rare case of pRCC presenting as a renal abscess. Awareness of this association between pRCC and a renal abscess, as infrequent as it may be, is essential for the urologist as it highlights the need for abscess wall biopsy and appropriate histological analysis to prevent misdiagnosis.

\section{Author Disclosure Statement}

Authors report no competing financial interests.

\section{REFERENCES}

1. Olson E, Asmussen T, Vicca A, Osborn D. A case report of renal abscess caused by Salmonella virchow phage type 1 associated with a papillary renal cell carcinoma. J Infect. 1999;38(1):5657.

2. Kumar V, Nanavati S, Ortiz L, Komal F, Singhal M. Papillary Renal Cell Carcinoma Presenting as a Perinephric Abscess. J Clin Diagn Res. 2018;12(4):OD01-OD03.

3. Mavuduru R, Bora G, Panwar P, Kakkar N. Papillary renal cell carcinoma with abscess formation: A report of three cases. Indian J Urol. 2018;34(1):79.

4. Ramos J, Aguado J, Pilar G, Ales J, Soriano F. Clinical Spectrum of Urinary Tract Infections Due to Nontyphoidal Salmonella Species. Clin Infect Dis. 1996;23(2):388-90. 